\title{
Fluid Flow and Mixing in a Six Strand Billet Caster Tundish: A Parametric Study
}

\author{
Pradeep K. JHA, Sukanta K. DASH and Sanjay KUMAR \\ Indian Institute of Technology, Kharagpur, Kharagpur, 721 302, India. E- mail: sdash@mech.iitkgp.ernet.in \\ (Received on April 16 2001; accepted in final form on August 9, 2001)
}

\begin{abstract}
Mixing phenomena in a six strand billet caster tundish has been studied by numerically solving the Navier Stokes equations along with the species concentration equation in a boundary fitted coordinate system comprising the geometry of the tundish. The solution of the species concentration equation has been utilized to compute the mix, dead and plug volume of the tundish under different flow conditions. The numerical procedure and solution algorithm has been first verified by comparing the numerically obtained residence time distribution curve, which agree well with that of the experiments done for a single strand bare tundish by Singh and Koria. ${ }^{44)}$ It has been observed that the ratio of the mix to dead volume for the six strand tundish has a maximum value for a particular position of the outlets. At that particular position of the outlets (where mixing is best), an APB is placed on the bottom of the tundish surrounding the incoming inlet jet and the height of the APB has been varied to see the effect on mixing in the tundish. It has been observed that the ratio of mix to dead volume further increases with the use of APB and attains a peak value after which it decreases with the increase of the height of the APB signifying the existence of an optimum APB height. At this optimum height of the APB, the shroud immersion depth was made to change from 0 to $400 \mathrm{~mm}$. It was also observed that there exists an optimum immersion depth of the shroud where the ratio of mix to dead volume still attains another peak signifying still better mixing. However, increasing the immersion depth to higher values spoils mixing significantly.
\end{abstract}

KEY WORDS: billet caster tundish; mixing; mean residence time; advance pouring box; shroud immersion depth.

\section{Introduction}

The growing demands on quality in steel products during the past couple of decades have made continuous casting of steel a widely used and an important step in the manufacture of steel. Considering this, increasing relevance was given to the tundish in latent years, becoming from a simple liquid steel distributor to a continuous metallurgical reactor where it is possible to clean the steel, or at least not to decrease the cleanliness. ${ }^{1)}$ The inclusion floatation and their removal can be maximized if the melt flow can be so modified as to result in having minimum stagnant volume conditions and optimum circulations in the tundish. ${ }^{2}$

There have been many researches reported on the benefits of flow control devices, ${ }^{3-7)}$ dams and weirs being the most common among them. Although some benefits in flow control were clearly observable, it was soon realized that the formation of dead volumes in the lee ward side of these flow control devices may spoil the overall residence time and hence mixing in the tundish. More recently efforts have been made to decrease heavy splashes during start up operations of casting sequences, ladle change operations and wear rate of tundish refractories. Elimination of the pick up of oxygen and nitrogen from the surrounding air through the turbulent zone, formed near the incoming liquid jet delivered by the submerged entry nozzle (SEN), has been an- other objective. ${ }^{8)}$ All this has been successfully achieved using a new kind of flow control device, better known as turbulence inhibitors ${ }^{9,10)}$ or Advanced Pouring Box (APB). These devices are a kind of an impact pad mostly with lips and interiors with right angles and squared or circular cross sections.

Even though a lot of studies on flow behavior in tundish has been carried out using physical modeling ${ }^{1-26)}$ as well as mathematical modeling, ${ }^{16,26-32)}$ very little information is available on multi-strand billet \& bloom casters taking the overall mixing phenomena in it as a whole. Madias et al. ${ }^{33)}$ have studied the effect of APB on mixing in a multi-strand tundish in an experimental work. They have found that the APB in general helps to increase mean residence time, plug volume and decrease dead volume. Morales et al. ${ }^{8)}$ have numerically studied the effect of APB and baffles on mean residence time and found that the mean residence time has increased along with the plug volume because of the use of APB and baffles. However, both these authors have not studied the height of APB on mixing extensively, and moreover no literature have been found to describe the effect of positions of outlet on mixing and the effect of shroud immersion depth on the same. Therefore, it has been intended to study the effect of these geometrical parameters on mixing as a whole in multi-strand tundish. It has been felt that just by changing the positions of the outlets there may be 
benefit in terms of gaining better mixing in the tundish as discussed in Sec. 3.2 rather than putting dams and weirs. It should be noted here that fixing up dams and weirs in a tundish is a much involved job rather than changing outlet positions, which seem to be rather easier. Moreover on the leeward side of the dams and weirs, there will normally be dead zones which rather spoils mixing in the tundish. So this has been tried in the present work and in addition to this an Advanced Pouring Box (APB) has been put around the inlet jet where it impinges on the bottom plane so that the resulting flow may create better mixing in the tundish. The effect of the height of the APB brings in interesting variations to the flow field and also to the mixing in general. The effect of the shroud immersion depth makes the flow field still benign to mixing, which is normally not found in literature in the present form. So the present study is an effort in this direction.

\section{Physical Description of the Problem}

The geometry of the billet caster tundish is shown in Fig. 1a along with the three outlets. The tundish is shown half because of the symmetry about the inlet plane. The depth of the tundish and the bottom pad are 572 and $280 \mathrm{~mm}$ respectively with the size of the inlet as $25 \mathrm{~mm} \times 50 \mathrm{~mm}$ and all other dimensions are shown in a plan view in Fig. 2, which completes the detail geometrical description of the industrial size tundish taken for the mathematical simulation. In Fig. 1c the arrangement of the APB (Advanced Pouring Box) is shown along with the grid lines and Fig. 1b shows the boundary fitted grid lines in the whole tundish. Figure 1d shows the shroud immersion depth along with the placement of APB. It is to be noted that the outlets $(15 \mathrm{~mm} \times$ $15 \mathrm{~mm}$ ) are drilled through the bottom pad at the positions shown and they have a length of $280 \mathrm{~mm}$ as the thickness of the bottom pad. The positions of the outlets are measured from the bottom wall and the exact locations of the outlets are shown in Fig. 2. At position-1 the outlets are placed at $50 \mathrm{~mm}$ away from the wall while at position-3 the outlets are at $300 \mathrm{~mm}$ away from the wall. While the outlets are at position 2 the APB $(300 \mathrm{~mm} \times 300 \mathrm{~mm}$ in $\mathrm{X}$ and $\mathrm{Y})$ is placed under the inlet jet and the height of the APB is varied to see the effect of mixing in the tundish. At a particular height of the APB the shroud immersion depth is varied to compute the effect of mixing in the tundish. Mixing in the tundish is studied by injecting a dye through the inlet stream for a very small time interval and then computing the mass concentration of the dye in the entire tundish as a function of time. It is intended to compute the ratio of mixed to dead volume and the mean residence time in the tundish, which are regarded as the main parameters for deciding the effective utilization of the tundish volume. Also the response of the dye at all the outlets are monitored which in turn help to compute the mixed and dead volume as well as the mean residence time ${ }^{20-22)}$ defined by Eq. (7).

\subsection{Mathematical Formulation and Assumptions}

The flow field in the tundish is computed by solving the mass and momentum conservation equations in a boundary fitted coordinate system along with a set of realistic boundary conditions. The tundish boundary does not conform to a

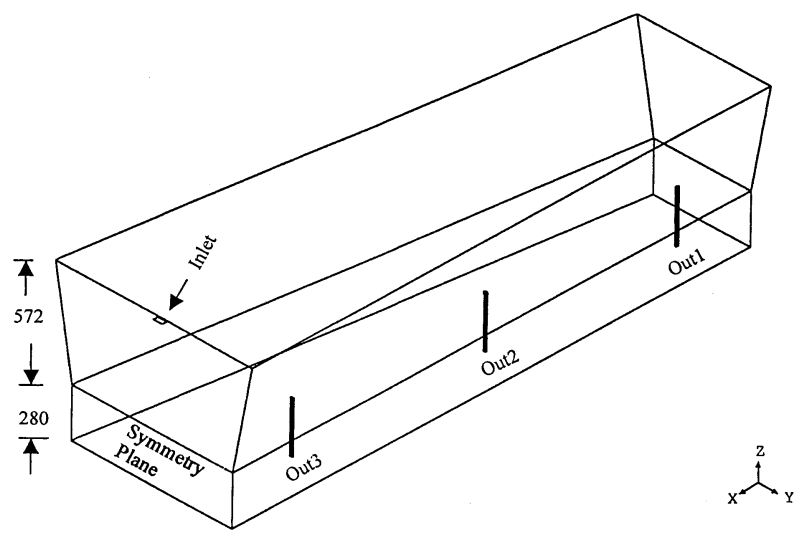

Fig. 1a. Geometry of the six strand billet caster tundish about the symmetry plane with the inlet and outlets drilled through the bottom pad.

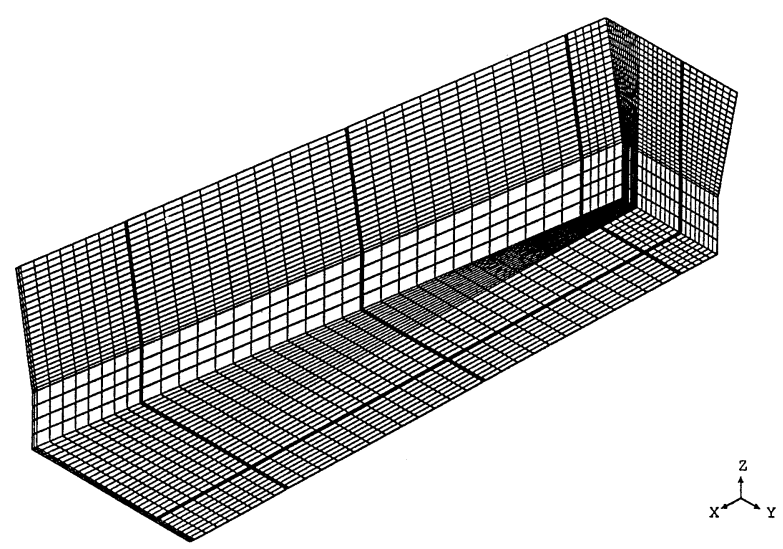

Fig. 1b. The billet caster tundish with the boundary fitted grid lines shown on the outermost surfaces.

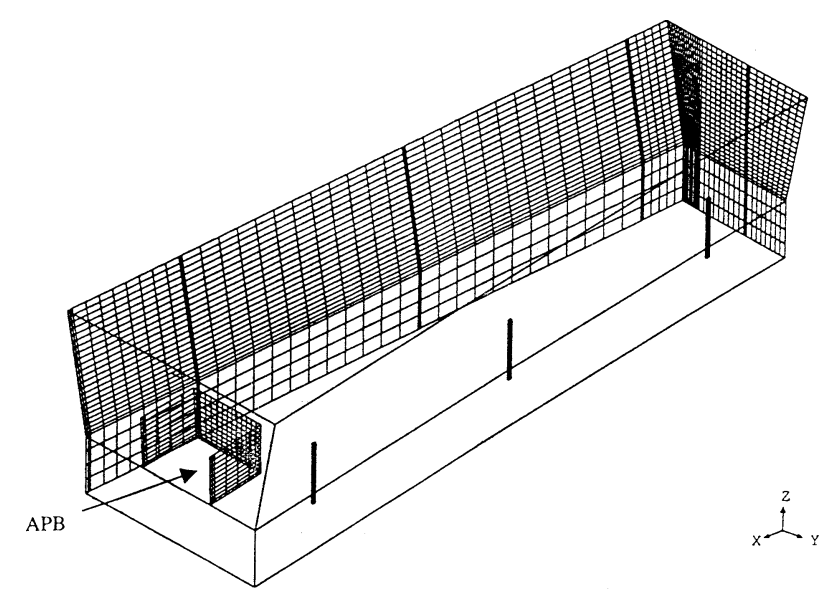

Fig. 1c. Boundary fitted grid lines showing the outer surfaces along with the advance pouring box (APB) placed on the bottom plane of the tundish.
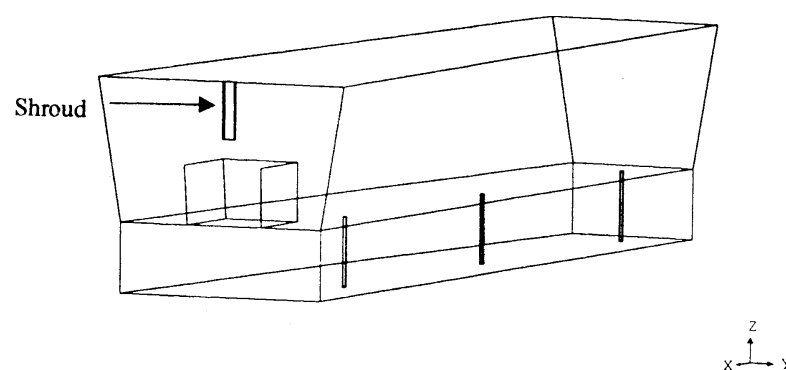

Fig. 1d. Geometry of the tundish (about the symmetry plane) with the APB placed on the bottom plane along with the shroud. 


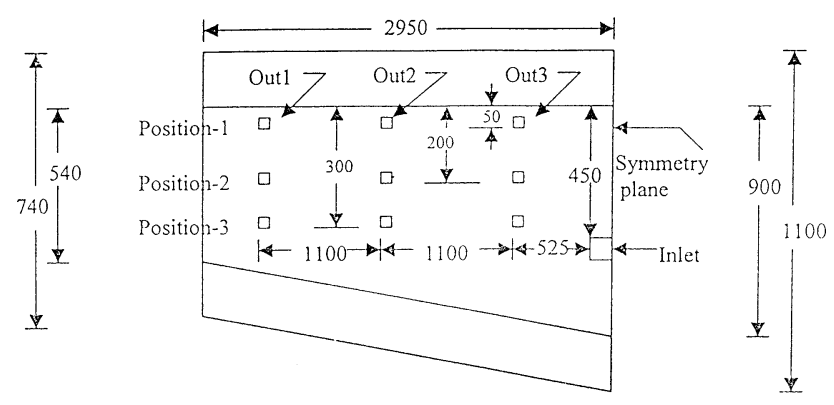

Fig. 2. Top view of the tundish about the symmetry plane with the locations of the outlets at the bottom plane. (All dimensions are in $\mathrm{mm}$.)

regular Cartesian system, because it is an inclined wall delta shape tundish, so the use of BFC has been made to solve all the conservation equations. The species continuity equation is solved in a temporal manner to capture the local variation of the concentration of the dye in the tundish. The free surface of the liquid in the tundish was considered to be flat and the slag depth was considered to be insignificant. With these two assumptions the flow field was solved with the help of the following equations (in tensorial form) with a built in $k-\varepsilon$ turbulence model ${ }^{34-36)}$ because the flow field is normally turbulent in the tundish. The effect of natural convection is neglected while computing the velocity field in the tundish because the ratio ${ }^{37)}$ of $\mathrm{Gr} / \mathrm{Re}^{2}=0.044(\Delta T)$, is much less than unity, where $\Delta T$, the driving force for natural convection, is the temperature difference between the liquid steel at the top free surface of the tundish to that of the bulk temperature of the liquid steel at that cross sectional plane.

Governing Equations

Continuity

$$
\frac{\partial}{\partial x_{i}}\left(\rho U_{i}\right)=0
$$

Momentum

$$
\frac{D\left(\rho U_{i}\right)}{D t}=-\frac{\partial p}{\partial x_{i}}+\frac{\partial}{\partial x_{j}}\left[\mu\left\{\frac{\partial U_{i}}{\partial x_{j}}+\frac{\partial U_{j}}{\partial x_{i}}\right\}-\overline{\rho u_{i} u_{j}}\right] \ldots .
$$

Turbulent kinetic energy

$$
\frac{D(\rho k)}{D t}=D_{k}+\rho P-\rho \varepsilon
$$

Rate of dissipation of $k$

$$
\frac{D(\rho \varepsilon)}{D t}=D_{\varepsilon}+C_{1} \rho P \frac{\varepsilon}{k}-C_{2} \frac{\rho \varepsilon^{2}}{k}
$$

Concentration

$$
\frac{\partial}{\partial t}(\rho C)+\frac{\partial}{\partial x_{i}}\left(\rho u_{i} C\right)=\frac{\partial}{\partial x_{i}}\left(\frac{\mu}{\sigma_{\mathrm{c}}} \frac{\partial C}{\partial x_{i}}\right)
$$

Where,

$$
\overline{u_{i} u_{j}}=\frac{2}{3} k \delta_{i j}-v_{\mathrm{t}}\left(\frac{\partial U_{i}}{\partial x_{j}}+\frac{\partial U_{j}}{\partial x_{i}}\right)
$$

$$
\begin{gathered}
v_{\mathrm{t}}=0.09 \frac{k^{2}}{\varepsilon} \\
D_{\phi}=\frac{\partial}{\partial x_{j}}\left[\left(\mu+\frac{\mu_{\mathrm{t}}}{\sigma_{\phi}}\right) \frac{\partial \phi}{\partial x_{j}}\right] \\
P=-\overline{u_{i} u_{j}} \frac{\partial U_{i}}{\partial x_{j}}
\end{gathered}
$$

\section{Constants}

$$
C_{1}=1.44, \quad C_{2}=1.92, \quad \sigma_{\mathrm{c}}=1.0, \quad \sigma_{k}=1.0, \quad \sigma_{\varepsilon}=1.3
$$

\subsection{Computation of Mixed and Dead Volume $e^{10,38)}$}

Theoretical mean residence time:

$$
\tau=(\text { Volume of tundish }) /(\text { Volumetric flow rate })
$$

Actual mean residence time:

$$
t_{\mathrm{r}}=\frac{\sum C_{\mathrm{av}_{i}} t_{i}}{\sum C_{\mathrm{av}_{i}}}, \quad i=1,2,3 \text { (for the three outlets) } \ldots
$$

Average break through time:

$$
t_{\mathrm{p}}=\text { First appearance of tracer at the exits }
$$

(time is averaged because of multi exits).

Fraction of dead volume: $\quad V_{\mathrm{d}} / V=1-t_{\mathrm{r}} / \tau$.

Fraction of plug volume: $\quad V_{\mathrm{d}} / V=t_{\mathrm{p}} / \tau$

Fraction of mixed volume: $\quad V_{\mathrm{m}} / V=1-V_{\mathrm{p}} / V-V_{\mathrm{d}} / V \ldots$

\subsection{Boundary Conditions}

Boundary conditions can be well visualized with reference to Fig. 1a. The symmetry plane is given a symmetry boundary condition, which implies a zero gradient condition for all variables normal to that plane. The walls were set to a no slip condition and the turbulent quantities were set from a log law wall function. At the inlet the velocity of the incoming jet was set to a prescribed value of $1.4 \mathrm{~m} / \mathrm{s}$ (1.61 ton/min of liquid steel) with a turbulent intensity of $2 \%$. The top surface of the tundish was taken to be a free surface where zero shear stress condition was applied according to references. ${ }^{6,29,39)}$ The bottom of the tundish was treated as a wall where no slip conditions were used for the velocity. At the outlets a fixed pressure of 1 atm was applied. The wall of the tundish was considered to be impervious to the dye, so a zero gradient condition for the dye was used on the walls. At the outlet and at the free surface also zero gradient conditions for the dye was used. ${ }^{39,40)}$ At the inlet the concentration of the dye was kept to 1 till $5 \mathrm{sec}$ after which the concentration was to kept to zero. Five seconds is normally very small compared to the mean residence time of the tundish so the influx of the dye during its travel is not likely to change the local velocity field because the mass influx of the dye is also very small. ${ }^{41)}$

\subsection{Method of Solution}

The set of partial differential equations, Eqs. (1) to (5), were solved with the help of above boundary conditions numerically in a finite volume technique using the educational 
version of the CFD software Phoenics. ${ }^{42)}$ The partial differential equations were integrated over a control volume to find out the fluxes (of mass and momentum as well as that of the dye) through all the faces and the flux balance is made over all the control volumes which yield a set of linear algebraic equations. The set of algebraic equations are solved by tridiagonal matrix (TDM) method ${ }^{43)}$ for momentum and by a whole field solver, taking one from the family of conjugate gradients, for the pressure correction equation. The species continuity equation is solved at each and every time step using the TDM matrix method once the steady state solution for the momentum equations are obtained. The solutions are said to have converged when the whole field normalized residuals for each of the velocity components and mass fall below unity. A false time step relaxation of 0.5 was used for all the variables for faster convergence. Control volumes $(\mathrm{CV})$ of $45 \times 34 \times 27(X \times Y \times Z)$ were used for the computation. By changing the control volumes to $58 \times 40 \times 35$, it was observed that the changes in the mixed and dead volumes were less than $0.2 \%$. So a lower CV was chosen for the computation. A separate validation of the RTD with the experimental observation of Singh and Koria ${ }^{44)}$ will be presented in the results and discussions. From the temporal variation of concentration the actual mean residence time and all other times are found out by simple integration after which the ratio of mixed to dead volume could be found out. For the computation, the density of liquid steel was taken to be $7100 \mathrm{~kg} / \mathrm{m}^{3}$ all through the volume and the kinematic viscosity ${ }^{10}$ to be $0.913 \times 10^{-6} \mathrm{~m}^{2} / \mathrm{s}$.

\section{Results and Discussions}

The flow field in the six strand billet caster tundish has been obtained by solving the Navier Stokes equations numerically and then the tracer dispersion was computed by injecting some dye into the inlet. From the tracer dispersion curve the mixed volume and the dead volume were computed as per Eqs. (9), (10) and (11). The analysis of mixing will be done with respect to the ratio of mixed to dead volume for different geometrical positions of the outlet, height of the APB and for different shroud immersion depth. Mixed volume and dead volumes are direct indices of mixing in a tundish. If the mixed volume is large that means more of the tundish volume is utilized in mixing the fluid. In a similar way it can be told that if the dead volume is low then most of the volume of the tundish is utilized by the fluid for mixing. So a ratio of mix to dead volume ${ }^{45)}$ and the mean residence time are better parameters to describe the mixing in the tundish as a function of other geometrical parameters. In the present study the effect of outlet positions, height of the APB and the shroud immersion depth on mixing has been carried out. So the presentations of the result will be done in the same sequence preceded by a validation of the result with an experimental observation.

\subsection{Validation of RTD}

Experimental observation on RTD for a six-strand billet caster tundish is not available in the literature, where a full comparison of the computed RTD can be done. So it was felt that before proceeding to the actual computation for an

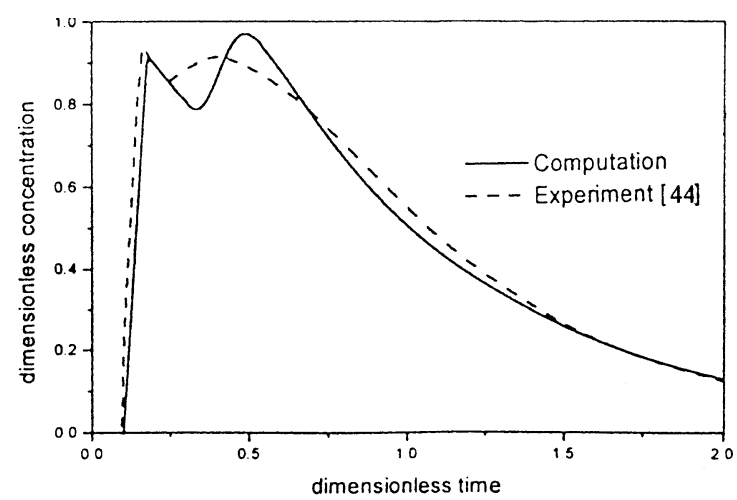

Fig. 3. Comparison of RTD for a bare tundish with one outlet, with that of the present computation.

industrial size tundish a validation of the computational method should be done at least with some available experiments. Singh and Koria ${ }^{44)}$ have done an experiment for a bare tundish with one inlet and one outlet. With their dimensions we carried out the computation for the velocity field and the concentration field with a grid of $66 \times 27 \times 30$ $(X \times Y \times Z)$. The RTD curve obtained from the present computation has been shown in Fig. 3 along with the experimental observations of Singh and Koria. It can be seen that the RTD curve has two peaks in the experiment and the same has been obtained through the computations also. The first peak value matches with that of the experiment, but the second peak value does not match well. The matching seems to be quite satisfactory everywhere leaving aside the second peak where the matching does not seem to be that good. The grids were also made finer in the outlet portion to capture the observed differences in the concentration near the second peak between the experiment and computation. However grid sensitivity does not make any difference to the reported computational curve. Considering a single point eddy viscosity turbulent model that has been used in the present computation, the matching between the experiment and the computation seems to be quite satisfactory. However, an idea of the computational grid size has been made from this comparison, which can be used to predict result closer to experimental observation.

\subsection{Effect of Outlet Positions}

The effect of outlet positions on the RTD for the bare tundish has been shown in Figs. $4 \mathbf{a}$ to $\mathbf{4 c}$ in terms of dimensionless concentration versus time. The dimensionless concentration is obtained by dividing the concentration $C_{i}$ by the quantity $Q / V$ for the tracer, ${ }^{40)}$ where $Q$ equals the amount of the tracer added and $V$ stands for the volume of the tundish. The dimensionless time is obtained by dividing the actual time with $\tau$ (theoretical mean residence time). When the outlets are at position-1, 2 or 3, their distances from the inner wall can be read from Fig. 2. It can be seen that when the outlets move away from the wall (move nearer to the inlet) the peak in the concentration for out3 decreases significantly. The peaks in the other two outlets, namely out 2 and out 1 suffer minor change. A very sharp peak in the concentration curve signifies very poor mixing. This happens because the fluid travel straight towards the outlets once they impinge on the tundish bottom, without getting any chance to mix with the surrounding fluids. 


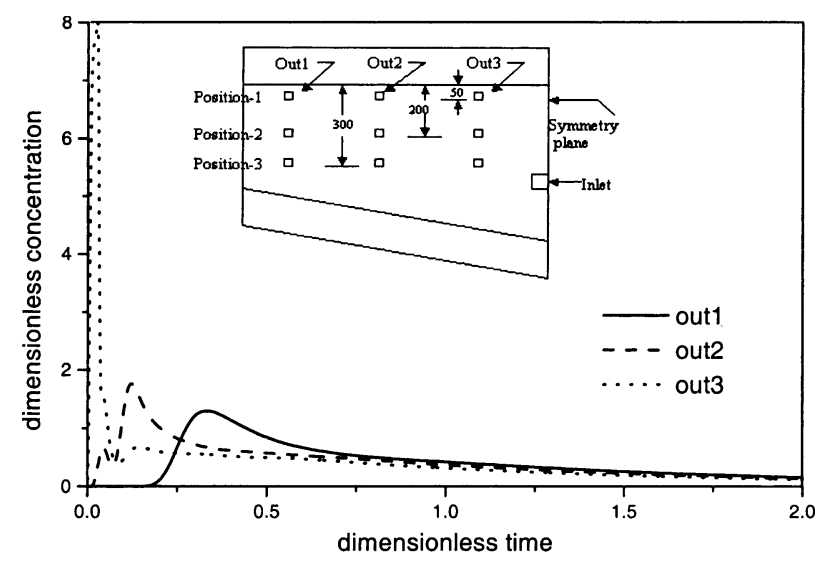

Fig. 4a. Variation of concentration with time for the outlets at position-1 for the bare tundish.

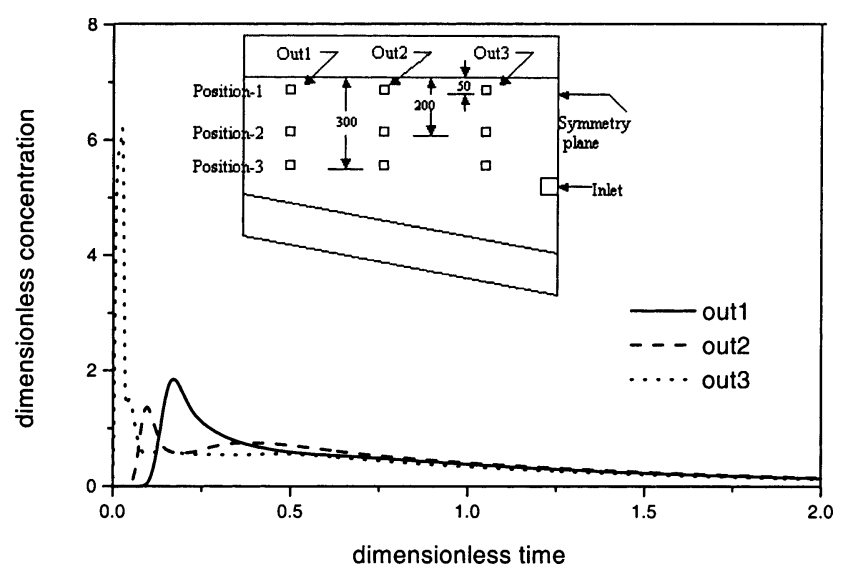

Fig. 4b. Variation of concentration with time for the outlets at position-2 for the bare tundish.

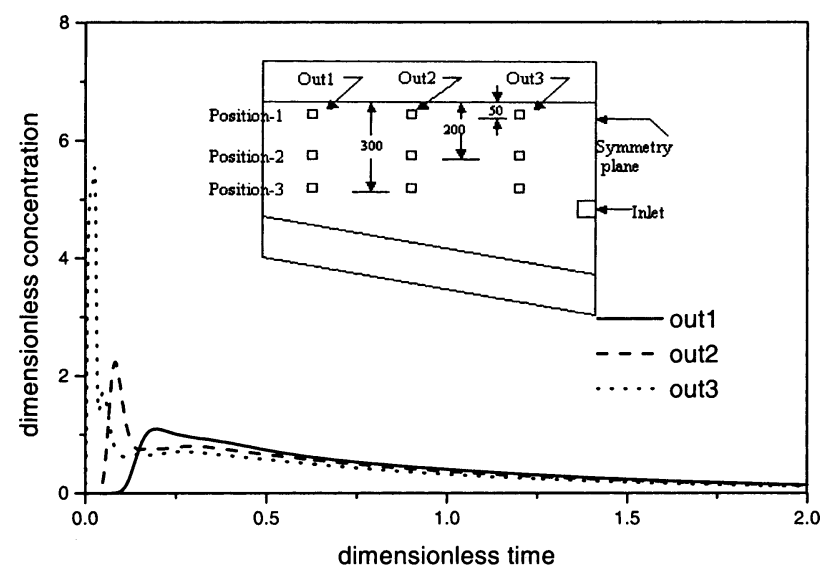

Fig. 4c. Variation of concentration with time for the outlets at position-3 for the bare tundish.

From Fig 4 it is difficult to conclude (between positions 2 and 3) which position of outlets is better in terms of mixing, although position 1 is seen to be out rightly bad.

Figures 5a to $\mathbf{5 c}$ show the velocity field passing through three vertical cross sectional planes containing the outlets when they are at positions 1, 2 and 3. It can be seen from the vector plot that while the outlets are at position- 2 mixing is better compared to all other positions. Figure $5 \mathrm{~b}$ shows strong recirculation in the gap between out 3 and out 2 and week recirculation between the last two outlets (out2

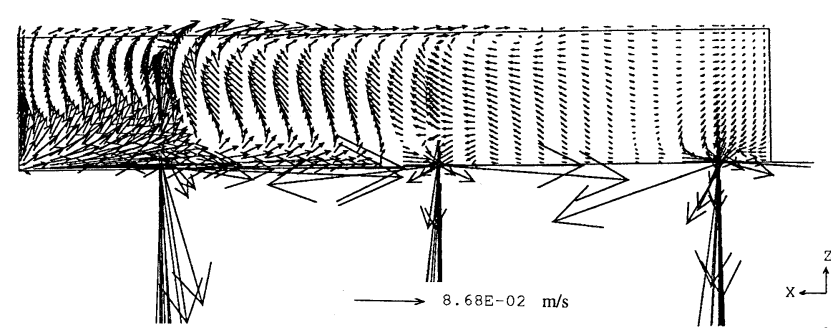

Fig. 5a. Velocity field through a vertical cross sectional plane passing through all the outlets at position-1 (bare tundish).

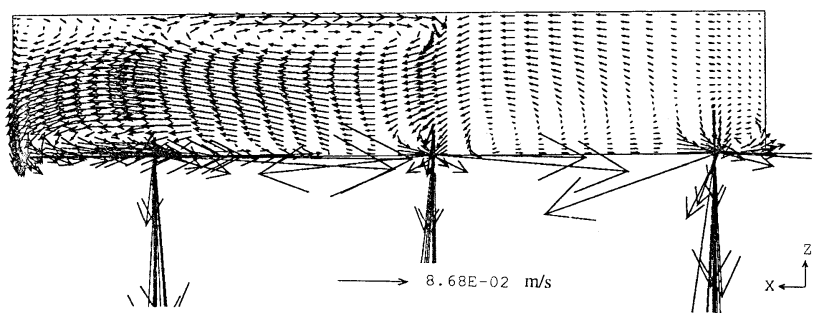

Fig. 5b. Velocity field through a vertical cross sectional plane passing through all the outlets at position-2 (bare tundish).

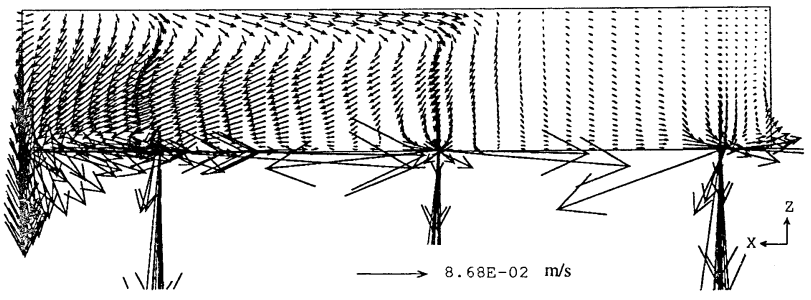

Fig. 5c. Velocity field through a vertical cross sectional plane passing through all the outlets at position-3 (bare tundish).

and out1). Figure 5c shows weaker recirculation compared to Fig. 5b in both the segments of the outlets, namely out3out 2 and out2-out1. So a vector plot just gives lot of idea about the mixing in a tundish, however this can not be used to predict the entire thing that takes place in a tundish. For this purpose the use of mixed and dead volume has been made. The plot of the ratio $V_{\mathrm{m}} / V_{\mathrm{d}}$ (ratio of mix to dead volume) has been shown in Fig $\mathbf{6 a}$ and the mean residence time in Fig. 6b as a function of the position of the outlets from the wall. It can be seen that when the outlets are at $200 \mathrm{~mm}$ from the wall the ratio of the mix to dead volume attains a peak and so also the mean residence time. This signifies that mixing is better at this position of the outlets and any other positions spoils mixing to some extent. When the outlets are closer to wall the impinging jet, which spread on the bottom plane of the tundish hit the wall and come back to be intercepted by the outlets. So the concentration curve shows peak values and mixing is normally not good. When the outlets are closer to the inlet the spreading liquid jet reaches the outlets faster which causes mixing to be again poor. So when the outlets are placed somewhere in between the wall and the inlet plane they show better mixing. Hence, an optimum location of the outlets exists where the ratio of the mix to dead volume and the mean residence time are found to be the highest. 


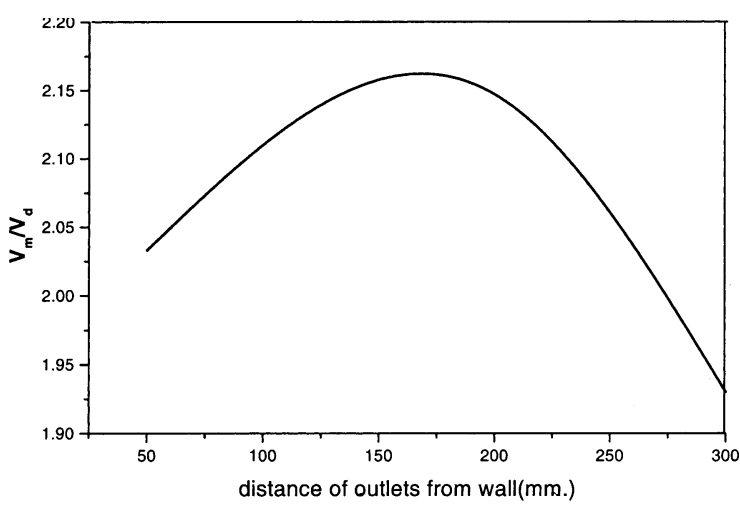

Fig. 6a. Variation of the ratio of mix to dead volume as a function of the distance of outlets from the wall.

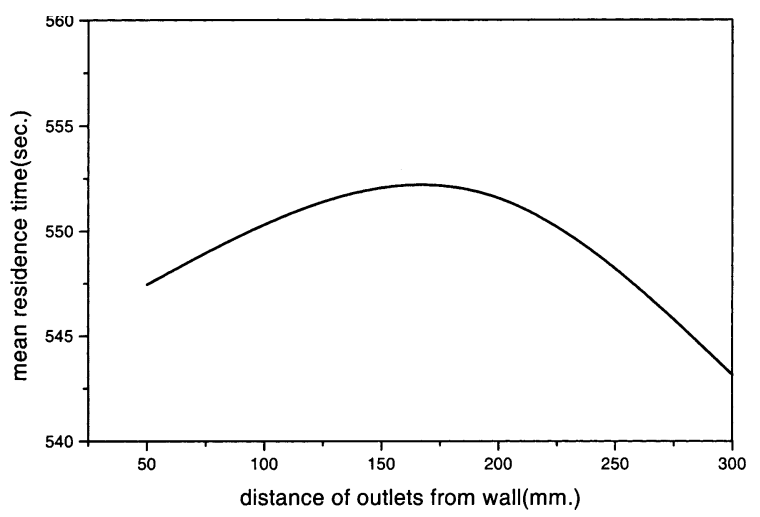

Fig. 6b. Variation of the mean residence time as a function of the distance of outlets from the wall.

\subsection{Effect of Height of APB}

It has been seen that mixing is better when the outlets are placed at position-2, i.e.; $200 \mathrm{~mm}$ away from the wall as shown in the Fig. 5b. While the outlets are placed at position-2 an APB is placed on the bottom of the tundish, which can surround the incoming inlet jet. By such a placement of the APB the velocity field on the bottom plane is arrested. The fluid particles are forced to move upward before they come towards the outlet. This way some mixing is enforced. But the open question remains as to the height of the APB. How high should be the APB so that mixing is best obtained? A sequential study on the variation of the height of the APB is done and the RTD curve for all the three outlets are shown in Figs. 7a to 7c for different heights of the APB. It can be seen that when the height of the APB is increased the peak value of the concentration for out 2 and out 1 has decreased. The peak value of the concentration for out3 (nearer to the inlet) has increased. This shows bad mixing for out 3 whereas for out 2 and out 1 mixing has improved. A conclusion can not be directly arrived from such a RTD curve regarding the overall improvement in mixing due to the change in height of the APB.

In Figs. 8a to $\mathbf{8 c}$ the velocity vectors are shown in a vertical cross sectional plane passing through all the outlets when the outlets are placed at position-2 but the height of the APB is varying from $90 \mathrm{~mm}$ (Fig. 8a) to $210 \mathrm{~mm}(8 \mathrm{~b})$ to $340 \mathrm{~mm}(8 \mathrm{c})$. It can be seen that when the APB has a height of $210 \mathrm{~mm}$ the recirculation at the outlet plane (Fig. $8 \mathrm{~b}$ ) is stronger compared to the case of the APB height of $90 \mathrm{~mm}$

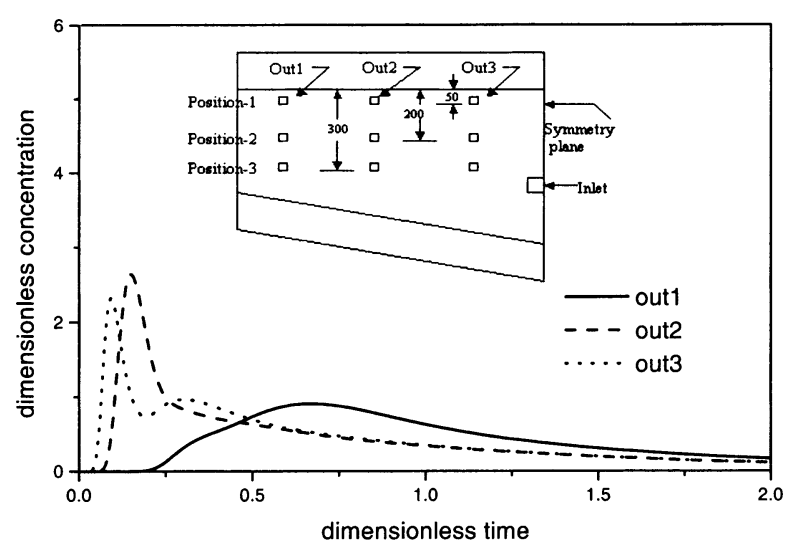

Fig. 7a. Variation of concentration with time for the outlets at position-2 with an APB height of $90 \mathrm{~mm}$.

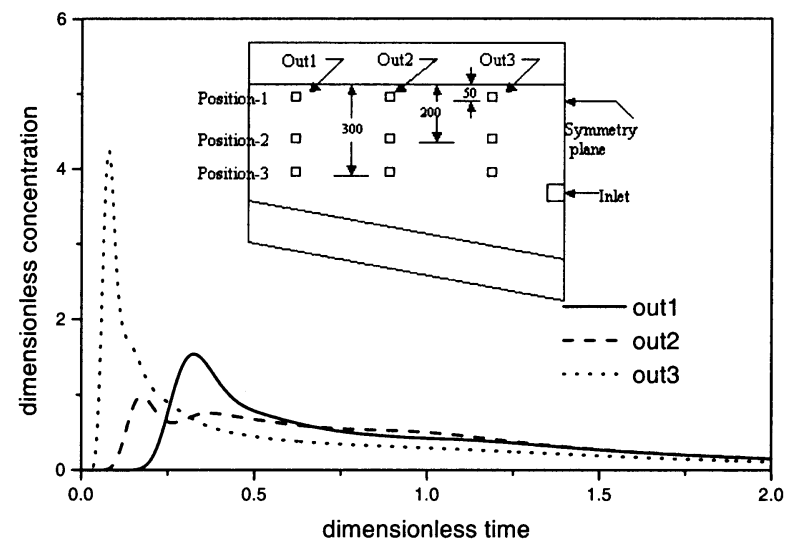

Fig 7b. Variation of concentration with time for the outlets at position-2 with an APB height of $210 \mathrm{~mm}$.

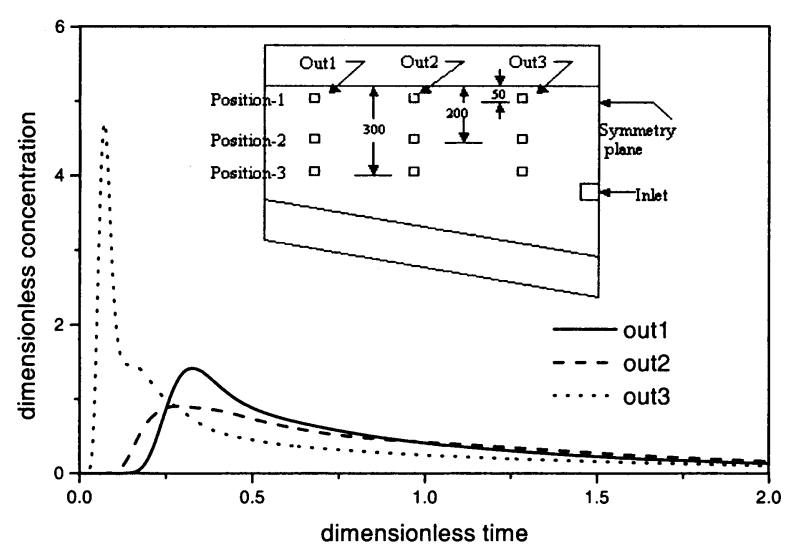

Fig. 7c. Variation of concentration with time for the outlets at position-2 with an APB height of $340 \mathrm{~mm}$.

(Fig. 8a). When the height of the APB has increased to $340 \mathrm{~mm}$ the recirculation has suffered a minor decrease compared to the case of APB height of $210 \mathrm{~mm}$. However, in order to see the mixing phenomena in the tundish as a whole the ratio of the mix to dead volume has been plotted as a function of the height of the APB in Fig 9a. Figure 9b shows the variation of mean residence time with the variation of the height of the APB. It can be observed that the ratio of mix to dead volume attains a maximum when the height of the APB has a value of $210 \mathrm{~mm}$. By placing the $\mathrm{APB}$ on the bottom plane of the tundish the inlet jet does 


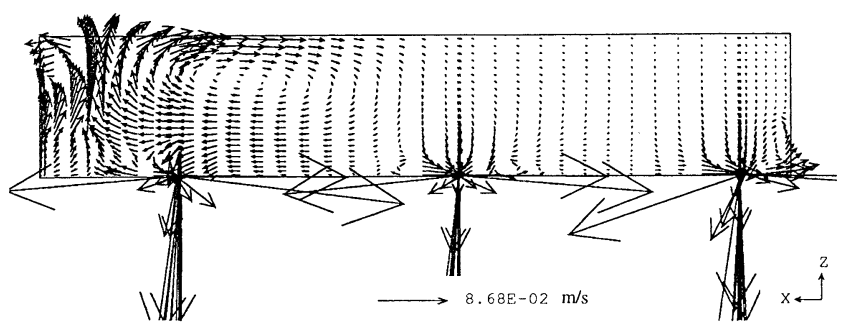

Fig 8a. Velocity field through a vertical cross sectional plane passing through all the outlets at position-2 for APB height of $90 \mathrm{~mm}$.

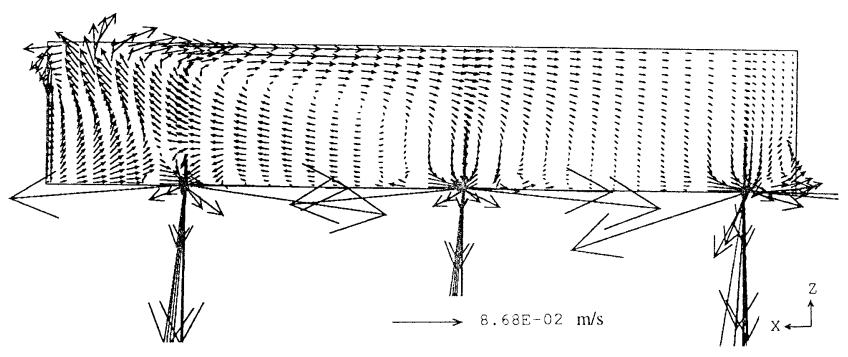

Fig. 8b. Velocity field through a vertical cross sectional plane passing through all the outlets at position-2 for APB height of $210 \mathrm{~mm}$.

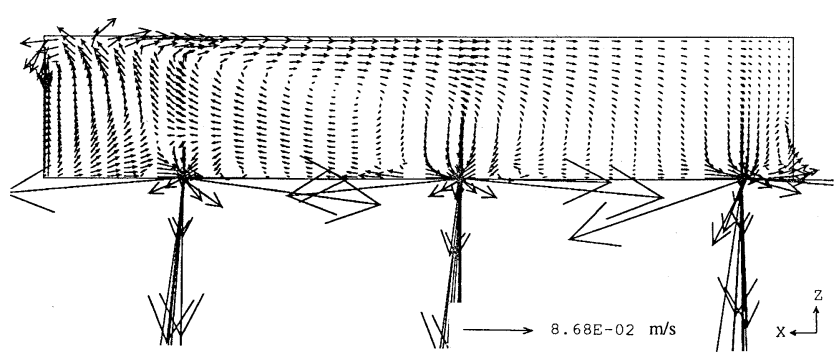

Fig. 8c. Velocity field through a vertical cross sectional plane passing through all the outlets at position-2 for APB height of $340 \mathrm{~mm}$.

not get a chance to flow directly to the outlets. So the jet travels upward first and then spreads into the tundish after which it can get a path to the outlets. This causes the hold up time to be more and hence the residence time increases and also the mixing. However, when the height of the APB increases beyond a certain height the plume coming out of the APB becomes mostly vertical or in other words it becomes another liquid jet of higher cross sectional area trying to reach the free surface of the melt. This upward moving jet although induces certain suction on its side but mostly travels to the surface and creates a surface flow failing to induce a global circulation in the tundish. Because of this, overall mixing falls in the tundish. The mean residence time also attains a maximum at a height of $210 \mathrm{~mm}$ of the APB. All other heights of the APB do not favor mixing the way a particular height does. So an optimum APB height exists for better mixing as has been found an optimum location exists for the outlets.

\subsection{Effect of Shroud Immersion Depth}

So far all the analysis has been done keeping the inlet at the top free surface of the tundish, as can be seen in Fig. 1a. However, from many practical reasons the inlet is normally

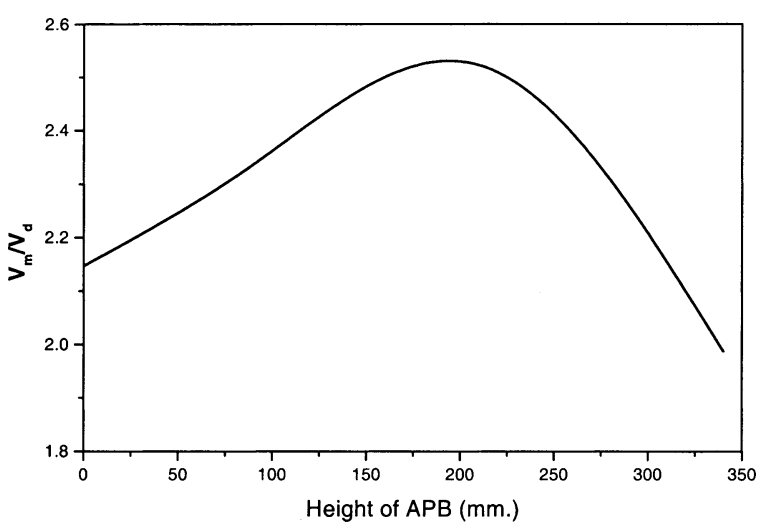

Fig. 9a. Variation of the ratio of mix to dead volume as a function of the APB height for outlets at position-2.

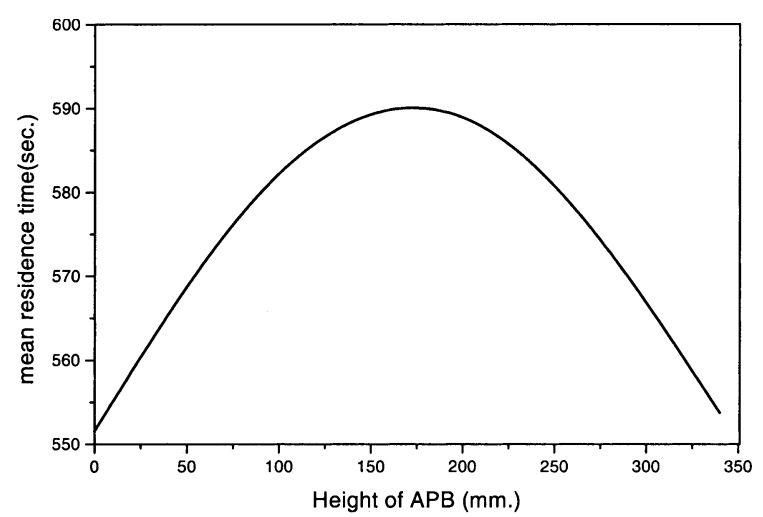

Fig. 9b. Variation of the mean residence time as a function of the APB height for outlets at position-2.

put inside the liquid bath, having some depth from the top free surface. This helps the liquid stream not to lose heat to the ambient and avoid entrapment of surrounding air at the point of impingement on the top free surface. Secondly the liquid jet can have stronger impact on the bottom pad, by coming closer to the pad, after which it can be diverted strongly by the help of the APB to other portion of the tundish causing better mixing. Therefore the use of a shroud has been made in the present study and its effect on mixing has been found out. The shroud immersion depth has been changed from 0 to $400 \mathrm{~mm}$ (measured from the top free surface) keeping the height of the APB at $210 \mathrm{~mm}$ (measured from the bottom pad) where the ratio of the mix to dead volume was found to be the highest. Again it should be noted here that, the outlets are at position-2 for this purpose because at this particular position the ratio of the mix to dead volume was found to be again the highest. At this particular position of the outlets and the height of the APB the variation of the ratio of the mix to dead volume is plotted as a function of the shroud immersion depth, shown in Fig. 10a. Figure 10b shows the mean residence time for this situation as a function of the shroud immersion depth.

It can be seen from Fig. 10a that the ratio of mix to dead volume decreases first as the shroud immersion depth increases and then again the ratio increases, attains a peak and then falls sharply again. When the shroud is introduced, the inlet plane of discharge goes in to the free surface and the recirculation in the fluid around the inlet plane falls a 


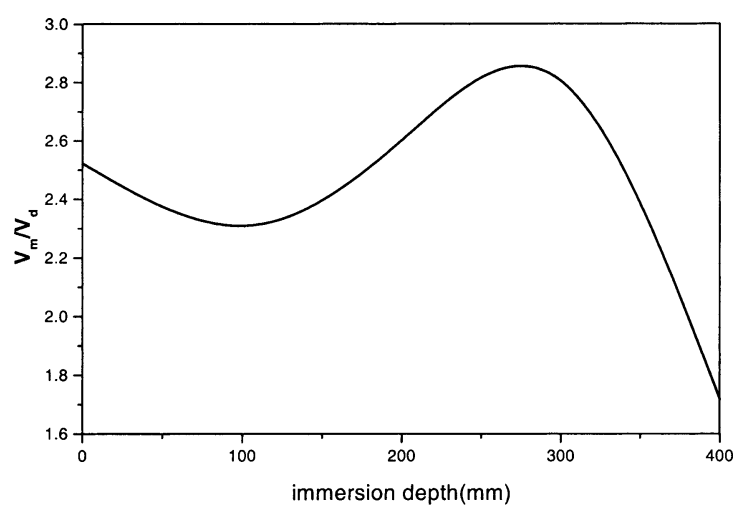

Fig. 10a. Variation of the ratio of mix to dead volume as a function of the shroud immersion depth for outlets at position-2 with APB height of $210 \mathrm{~mm}$.

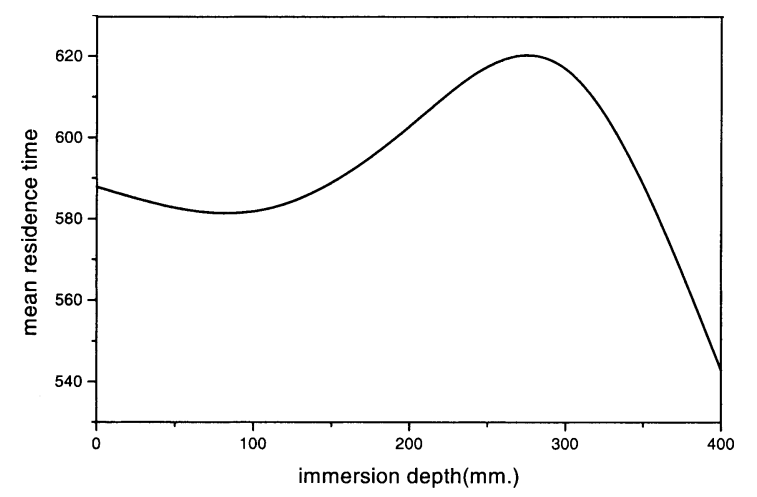

Fig. 10b. Variation of the mean residence time as a function of the shroud immersion depth for outlets at position-2 with APB height of $210 \mathrm{~mm}$.

little bit for which mixing deteriorates. This happens because the inlet stream does not get enough suction length as it moves deeper, down the free surface of the liquid. Such kind of observation has been made by Satish and Koria ${ }^{45}$ also, where they have mentioned the deterioration of mixing with shroud. However their study does not include the APB. Normally with the inclusion of an APB, the inlet jet, after striking the bottom pad gets a chance to rise along the walls of the APB where it can suck in the surrounding fluid towards it causing a larger recirculation zone in the tundish. So normally shroud is to be placed along with flow modifiers like APB. Figure 11a shows the velocity field on a cross sectional plane passing through the inlet with no shroud and with an APB height of $210 \mathrm{~mm}$. The shroud and APB are not shown in the figures for the sake of clarity of the velocity vectors. It can be seen from the vector plot that there is a large recirculation zone near the inlet plane due to the suction effect of the inlet jet. This suction effect falls a little the moment the inlet plane is dipped into the free surface of the liquid by the help of a shroud. The strength of the recirculation falls near the inlet plane and this can be seen very clearly in Fig. 11b where the shroud immersion depth has been increased to $100 \mathrm{~mm}$. The decrease in the strength of the vortex or the recirculation causes mixing to be poor so the ratio of mix to dead volume falls with the increase of the shroud immersion depth. When the strength of the vortex falls in the tundish the mean flow velocity towards the outlets increases so the fluid particles reach the

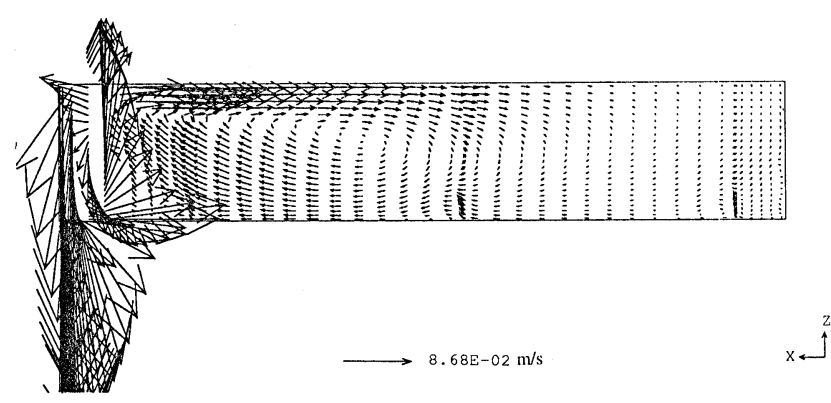

Fig. 11a. Velocity field through a vertical cross sectional plane passing through the inlet with APB height of $210 \mathrm{~mm}$ and no immersion depth for outlets at position 2.

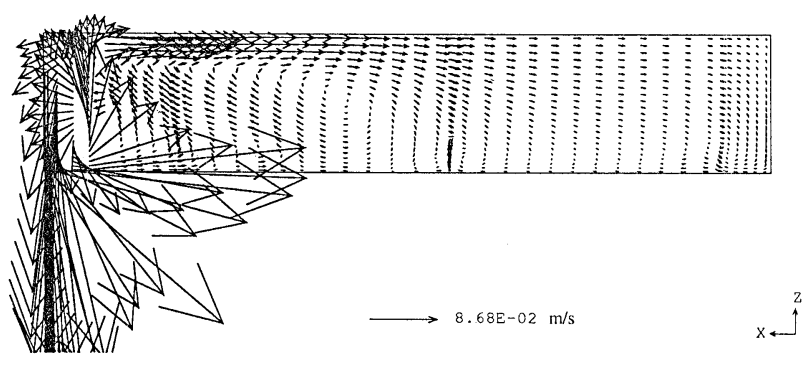

Fig. 11b. Velocity field through a vertical cross sectional plane passing through the inlet with APB height of $210 \mathrm{~mm}$ and immersion depth of $100 \mathrm{~mm}$ for outlets at position2.

outlets faster thereby causing the mean residence time to be lower. This can be seen from Fig. 10b where the mean residence time has fallen with the increase of the shroud immersion depth.

When the shroud immersion depth further increases the suction due to the inlet jet during its downward travel falls still further. But the rising plume from the APB becomes stronger and this rising velocity can be regarded as an upward jet but pretty large in size which can induce still suction of the fluid from the neighboring area of the APB once the jet has come out of the APB. As long as the jet is confined to the APB it can not suck any outside fluid towards it. So a suction of the fluid is created again due to the rising plume of the striking inlet jet. This suction can grow in strength and can induce stronger recirlulation in the inlet zone, which can again boost up mixing. This has happened when the shroud immersion depth has been increased to $200 \mathrm{~mm}$ from $100 \mathrm{~mm}$ (at which mixing seems to be lowest). The ratio of the mix to dead volume has again increased signifying better mixing. When the immersion depth has been still increased to $300 \mathrm{~mm}$ the suction has grown stronger due to the rising plume so also the strength of the recirculation, causing the mixing to be still better. This is why there is a peak on the ratio of the mix to dead volume again. However, from here onwards if the shroud immersion depth is still increased the shroud protrudes into the APB. With the shroud immersion depth at $400 \mathrm{~mm}$ the inlet jet is very close to the bottom pad. The inlet jet spreads on the bottom of the pad and tries to rise along the walls of the APB with very high velocity as because the impinging velocity of the inlet jet itself has gone up very high by bringing the inlet closer to the bottom pad (see Figs. 11b and $12 \mathrm{~b}$ ). The rising plume from within the APB has become also stronger and as a result the plume reaches the 


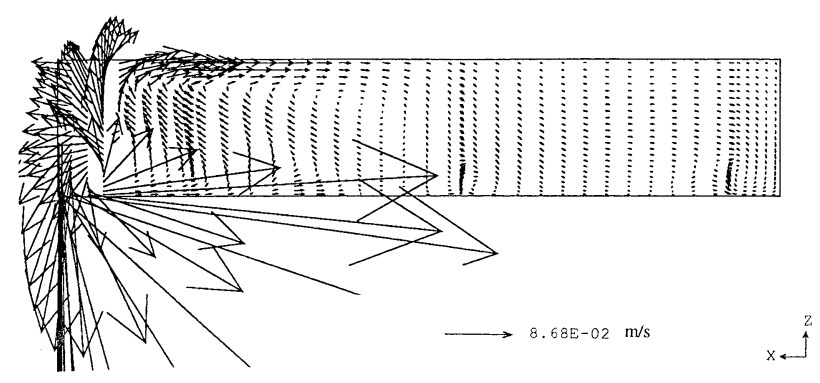

Fig. 12a. Velocity field through a vertical cross sectional plane passing through the inlet with APB height of $210 \mathrm{~mm}$ and immersion depth of $300 \mathrm{~mm}$ for outlets at position2.

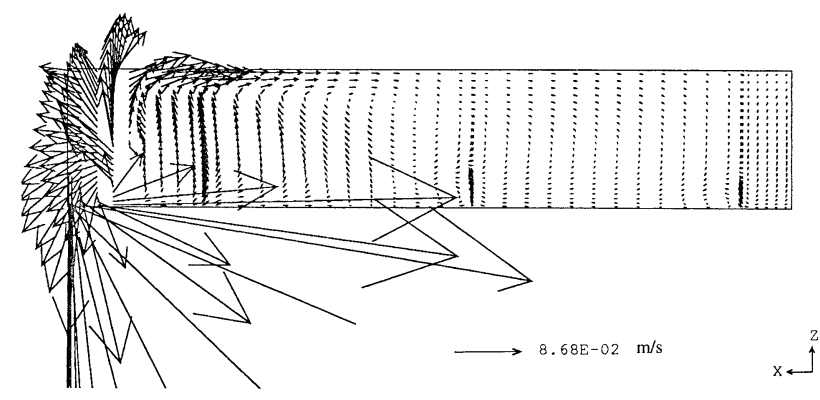

Fig. 12b. Velocity field through a vertical cross sectional plane passing through the inlet with APB height of $210 \mathrm{~mm}$ and immersion depth of $400 \mathrm{~mm}$ for outlets at position2 .

free surface of the liquid much faster compared to the case of an APB of height $300 \mathrm{~mm}$, after which it spreads on the free surface causing a lower recirculation in the vicinity of the free surface because the plume gets enough surface area to spread about. So the rising plume fails to induce a global recirculation in the tundish for which the mixing as a whole deteriorates in the tundish. In the case of the APB of height $300 \mathrm{~mm}$, the rising plume was not strong enough to reach the free surface, as a result it was able to suck the near by fluid in the tundish while coming out of the APB. So recirculation in the tundish near the APB was stronger which could set in some recirculation up to the second outlet out2, which results in a better global mixing. However, it should be noted that the velocity vectors through the inlet plane are used to describe the mixing phenomena in general in a tundish but the velocity field on this plane only, does not decide mixing solely in the tundish. It is just a representative field through which some insight can be gained to the mixing phenomena in a tundish where the flow field is really very complicated. While the flow field serves to give some idea about the mixing visually, but the computation of the ratio of mix to dead volume from the RTD curve gives a definite conclusive answer to the mixing quantitatively.

The velocity vector plot on a cross sectional plane passing through the inlet is shown in Fig. 12a with the shroud immersion depth as $300 \mathrm{~mm}$ and in Fig. 12b with the immersion depth as $400 \mathrm{~mm}$. It can be seen that the strength of the recirculation is stronger in case of a $300 \mathrm{~mm}$ shroud compared to the case of a $400 \mathrm{~mm}$ shroud. So the $400 \mathrm{~mm}$ shroud does not do any better trick as far as inducing a stronger recirculation is concerned near the inlet area. So the mixing gets affected severely and the mean residence time also. From this study it can be concluded that there exists an optimum shroud immersion depth for which the ratio of mix to dead volume and the mean residence time are the highest.

\section{Conclusions}

The mass, momentum and the species conservation equations have been solved numerically in a boundary fitted coordinate system comprising a typical industrial size tundish having a through put of $1.61 \mathrm{ton} / \mathrm{min}$. The ratio of the mix to dead volume and the mean residence time has been analyzed from the solution of the species conservation equation.

It has been found that there is an optimum location of the outlets (position-2) where the ratio of mix to dead volume has a maximum as well as the mean residence time, which signifies better mixing in the tundish.

There also exists an optimum height of the APB, of $210 \mathrm{~mm}$, where the ratio of mix to dead volume and the mean residence time has further maximum values.

It has been also found that there is an optimum shroud immersion depth for which the ratio of mix to dead volume and the mean residence time further attains another maximum values.

In this study it has been found that the maximum value of the ratio of mix to dead volume and the mean residence time has been increasing continuously with the optimum locations of the outlets and the optimum height of the APB and with the optimum shroud immersion depth.

\section{Acknowledgements}

The second author Dr. SKD, gratefully acknowledges the support of Alexander von Humboldt Foundation for donating him a high end $\mathrm{PC}$ on which the present computations were carried out along with the preparation of the manuscript.

\section{Nomenclature}

$C$ : Concentration of tracer

$C_{\mathrm{av}_{i}}$ : Average concentration of the tracer at outlet $i$, $(i=1,2,3)$

$k$ : Turbulent kinetic energy

$p$ : Pressure

$t:$ Time

$t_{\mathrm{r}}$ : Actual mean residence time of fluid in the vessel, Eq. (7)

$U:$ Velocity

$V$ : Volume of the tundish

$x$ : Coordinate for measure of distance

$\rho:$ Density of the fluid

$\mu$ : Co-efficient of viscosity

$v$ : Kinematic viscosity

$\overline{u_{i} u_{j}}:$ Average turbulent stress

$\varepsilon$ : Rate of dissipation of turbulent kinetic energy

$\sigma_{\mathrm{c}}$ : Turbulent Schmidt number

$\tau$ : Theoretical mean residence time, Eq. (6)

$\phi$ : $\quad$ Either $k$ or $\varepsilon$

Suffix

$i, j, k$ : Three Cartesian coordinate directions $x, y$ and $z$

d: Dead volume 


$$
\begin{array}{cl}
\mathrm{m}: & \text { Mixed volume } \\
\mathrm{p}: & \text { Plug volume }
\end{array}
$$

\section{REFERENCES}

1) A. McLean: Proc. Steelmaking Conf., ISS, Warrendale, PA, (1988), 3.

2) Y. He and Y. Sahai: Metall. Trans., 18B (1987), 81.

3) R. Ahuja and Y. Sahai: Proc. 24th Annual Conf. of Metallurgists, CIM, Canada, (1985), 124.

4) J. Tsukobura, I. D. Sommerville and A. McLean: 24th Annual Conf. of Metallurgists, CIM, Canada, (1985), 124.

5) Y. He and Y. Sahai: Acta Metall. Sin., 2 (1988), 93.

6) J. Szekely, O. J. Illegbusi and N. El-Kaddah: Phys. Chem. Hydrod., 9 (1987), 453.

7) S. Joo and R. I. L. Guthrie: Metall. Trans., 24B (1993), 755.

8) R. D. Morales, S. L. Ramirez, J. P. Ramos and D. Zacharias: ISIJ Int., 39 (1999), 455.

9) P. Rasmussen: Proc. 77th Steelmaking Conf., ISS, Warrendale, PA, (1994), 219.

10) D. Bolger and K. Saylor: Proc. 77th Steelmaking Conf., ISS, Warrendale, PA, (1994), 225.

11) D. J. Harris and J. D. Young: Continuous Casting, Vol. 1, ISS-AIME, Warrendale, PA, (1983), 99.

12) Y. Yoshii, Y. Habu, T. Nozaki, S. Itoyama, H. Nishikawa and T. Imai: Int. Conf. on Technology and Applications of HSLA Steels, ASM, Metals Park, OH, (1983), 377.

13) E. Martinez, M. Maeda, L. J. Heaslip, G. Rodriguez and A. McLean: Trans Iron Steel Inst. Jpn., 26 (1986), 724.

14) A. van der Heiden, P. W. Van Hasselt, W. A. de Jong and F. Blass: Proc. 5th Int. Iron and Steel Conf., Vol. 6, AIME, New York, (1986), 755.

15) N. A. McPherson: Metall. Plant Technol., 3 (1986), 40.

16) O. J. Illegbusi, J. Szekely, R. Boom, A. van der Heiden and J. Klootwijk: Proc. of W. O. Philbrook Memorial Symp. Conf., Toronto, Canada, (1988), 185.

17) O. J. Harris, L. J. Heaslip, K. E. O'Leary, R. W. Pugh and C. Jager: Proc. Int. Symp. on Direct Rolling and Hot Charging of Strand Cast Billets, Vol. 10, ed. by CIM, Perganian Press, (1988), 19.

18) F. Salvati, P. Tolve, M. Masala, E. Peisino and D. Broglio: Proc. 1st European Conf. on Continuous Casting, Vol. 2, AIM, Florence, (1991), 173.

19) P. Tolve, A. Praitoni and A. Ramacciotti: Steel Times, 3 (1987), 128.

20) J. Knoepke and J. Mastervich: Steelmaking Conf. Proc., Vol. 69, ISS-AIME, Warrendale, PA, (1986), 777.
21) F. Kemney, D. J. Harris, A. McLean, T. R. Meadowcroft and J. D. Young: Proc. 2nd Process Technology Conf., ISS-AIME, Warrendale, PA, (1981), 232.

22) Y. Sahai and R. Ahuja: Ironmaking Steelmaking, 13 (1986), 241.

23) S. Govindrajan, S. K. Ajmani, A. Chatterjee and T. Mukherjee: Proc. Int. Symp. on Modern Developments in Continuous Casting, New Delhi, (1988), 153.

24) S. Hintikka and J. Konttinen: Proc. 1st European Conf. on Continuous Casting, Vol. 2, AIM, Florence, (1991), 153.

25) L. Xintian, Z. Yaohe, S. Baolu and J. Weiming: Ironmaking Steelmaking, 19 (1992), 221.

26) J.-L. Yeh, W.-S. Hwang and C.-L. Chou: Ironmaking Steelmaking, 19 (1992), 501.

27) T. Debroy and J. A. Sychterz: Metall. Trans., 16B (1985), 497.

28) J. Szekely and N. El-Kaddah: SCAN INJECT IV, MEFOS, Lulea, Sweden, (1986), 14: 1.

29) K. H. Tacke and J. C. Ludwig: Steel Res., 58 (1987), 262.

30) J. M. Camplin, J. Herbertson, H. Holl, P. Whitehouse, R. I. L. Guthrie, J. W. Han and M. Hasan: Proc. 6th Int. Iron and Steel Cong., ISIJ , Tokyo, (1990), 207.

31) S. M. Lee, Y. S. Koo, T. Kang, I. R. Lee and Y. K. Shin: Proc. 6th Int. Iron and Steel Cong., ISIJ, Tokyo, (1990), 239.

32) S. Joo and R. I. L. Guthrie: Can. Metall. Q., 30 (1991), 261.

33) J. Madias, D. Martin, M. Ferreyra, R. Villoria and A. Garamendy: ISIJ Int., 39 (1999), 787.

34) D. B. Spalding: Int. J. Num. Math. Eng., 4 (1972), 551.

35) B. E. Launder and D. B. Spalding: Mathematical Models of Turbulence, Academic Press, London, (1972).

36) S. K. Dash: Int. J. Num. Meth. Heat Fluid Flow, 6 (1996), 37.

37) S. Lopez-Ramirez, R. D. Morales and J. A. Romero Serrano: Numer. Heat Transfer, Part A, 37 (2000), 69.

38) O. Levenspiel: Chemical Reaction Engineering, John Wiley \& Sons Inc., New York, (1972).

39) O. J. Illegbusi and J. Szekely: Ironmaking Steelmaking, 16 (1989), 110.

40) O. J. Illegbusi and J. Szekely: Steel Res., 59 (1988), 399.

41) J. Szekely and N. J. Themelis: Rate Phenomena in Process Metallurgy, John Wiley \& Sons Inc., New York, (1971).

42) D. B. Spalding: Mathematics and Computers in Simulation, XIII (1981), 267.

43) S. V. Patankar and D. B. Spalding: Numerical Heat Transfer and Fluid Flow, McGraw-Hill, New York, (1980).

44) S. Singh and S. C. Koria: ISIJ Int., 33 (1993), 1228.

45) S. Singh and S. C. Koria: Steel Res., 66 (1995), 294. 